\title{
Cori Cycle Activity in Man
}

\author{
Christine Waterhouse and Julian Keinson \\ From the Department of Medicine, University of Rochester School of Medicine \\ and Dentistry and the Department of Statistics, University of Rochester \\ College of Arts and Sciences, Rochester, New York 14620
}

\begin{abstract}
A в S T R A C T 12 subjects have been studied after an overnight fast with trace amounts of pyruvate- $3-{ }^{14} \mathrm{C}$ and glucose-6- ${ }^{14} \mathrm{C}$. Blood disappearance curves and incorporation of the pyruvate- $3-{ }^{14} \mathrm{C}$ label into blood glucose have been determined. By the use of transfer functions which allow processes with many different chemical steps to be examined as a unit, we have determined the per cent of pyruvate and presumably lactate which is regenerated into glucose. 8 of the 12 subjects showed that $7-23 \mathrm{mg} / \mathrm{kg}$ per $\mathrm{hr}$ are recycled, while 4 subjects fell well outside this range. Correlation of increased activity was not good with any demonstrated metabolic abnormality (diabetes or obesity), and it is suggested from clinical observation of the subjects that anxiety may play a role.
\end{abstract}

\section{INTRODUCTION}

Hepatic glucose output as calculated from arteriovenous (AV) difference and hepatic blood flow has been estimated at $6-7 \mathrm{~g} / \mathrm{hr}$ in normal man after an overnight fast (1). Considering this as total blood glucose production, this should include glucose derived from liver glycogen as well as all processes resulting in gluconeogenesis. The major substrates for gluconeogenesis are certain amino acids, lactate and pyruvate, and perhaps to a minor extent glycerol liberated from peripheral tissues. The magnitude of the Cori cycle (glucose $\rightarrow$ lactate $\rightarrow$ glucose) and its contribution to the process of gluconeogenesis are of inherent interest as well as of importance for the proper interpretation of glucose ${ }^{14} \mathrm{C}$ disappearance curves.

All data referable to quantitation of the Cori cycle analyses in man have been done by the method proposed by Reichard in $1963(2-4)$. This method is dependent on the randomization of the label from $C_{1}$ of glucose to carbons 2, 5, and 6 during the process of gluconeogenesis from pyruvate. In this method, it is assumed that there is instantaneous resynthesis and release into the blood of

Received for publication 23 December 1968 and in revised form 10 June 1969. glucose derived from lactate and pyruvate. The analysis itself also views glucose as distributed in a homogeneous pool within the body, thus neglecting the early portion of the glucose ${ }^{14} \mathrm{C}$ disappearance curve. Furthermore, the measurement of only one of the four randomized carbon atoms necessitates multiplication of the determined values found in glucose- 6 by a factor of 4 , thus magnifying any errors of the determination.

For the above reasons, it was decided to reexamine Cori cycle activity in a series of patients by another technique and furthermore to compare the disposal rate of glucose as determined by tracer technique with other values of hepatic glucose production. The current study determines the amount of labeled glucose present in blood at various time intervals after a single injection of pyruvate- $3-{ }^{i 4} \mathrm{C}$. The integrated response is then corrected for glucose- $6-{ }^{14} \mathrm{C}$ disappearance, enabling quantitation of the fraction of pyruvate recycled to glucose.

\section{METHODS}

Data on the subjects used in the study are presented in Table I. Subjects 1 through 7 had no organic disease with the exception that R. N. had inactive rheumatic heart disease and E. C. had inactive mild rheumatoid arthritis. It should be noted that subjects $3,4,5,6$, and 7 were all originally referred with the question of hypoglycemic attacks with symptoms of anxiety, tremulousness, and hunger. Thorough study never documented hypoglycemia, even when attacks were observed in these subjects and the symptoms were felt to be related to anxiety. We point out the unfortunate circumstance that only one of the control subjects was male.

All patients were hospitalized at the Clinical Research Center, and the basic dietary preparation was that of a high carbohydrate intake. After an overnight fast, $10 \mu \mathrm{c}$ of pyruvate- $3-{ }^{14} \mathrm{C}^{1}$ was given by a single injection technique. Arterial samples were obtained for blood disappearance at $2,3,4,5,6,16,30,60,90$, and $150 \mathrm{~min}$. Total radioactivity was determined from plasma on these samples by precipitating the protein and counting in Bray's solution. Additional

${ }^{1}$ Obtained from New England Nuclear Corp., Boston, Mass. and tested for purity by paper chromatography with scanning. Five different preparations were used for the entire study, and no injectable material was used which had been stored more than 3 months. 
TABLE I

Patient Data

\begin{tabular}{lclrl}
\hline I Patient & Age & Sex & Weight & \multicolumn{1}{c}{ Diagnosis } \\
\hline & $y r$ & & & \\
R. N. & 51 & F & 64.06 & Control subject \\
J. F. & 21 & M & 67.78 & Control subject \\
P. L. & 43 & F & 59.06 & Control subject \\
K. H. & 22 & F & 48.42 & Control subject \\
D. P. & 48 & F & 42.41 & Control subject \\
C. V. & 30 & F & 53.29 & Control subject \\
E. C. & 36 & F & 56.93 & Control subject \\
D. R. & 48 & F & 57.46 & Mild diabetes \\
J. S. & 80 & F & 74.74 & Diabetes \\
M. O. & 45 & F & 84.77 & Obesity \\
N. S. & 21 & F & 115.37 & Obesity, abnormal GTT* \\
V. S. & 49 & F & 191.96 & Obesity, abnormal GTT \\
\hline
\end{tabular}

* GTT, glucose tolerance test.

blood was taken usually at $5,15,30,60,90$, and $150 \mathrm{~min}$ and from these samples glucose was isolated as potassium gluconate (5) and assayed for radioactivity by suspension in thixin gel. On a separate day, glucose- $6-{ }^{14} \mathrm{C}$ was given as a single injection tracer and the blood disappearance of this was determined as previously described (6), as was the ${ }^{14} \mathrm{CO}_{2}$ excretion as determined at $30,60,90,150$, and $210 \mathrm{~min}$.

\section{RESULTS}

Glucose disappearance curves. In 8 of the 12 patients, the disappearance of labeled glucose from the blood was remarkably similar and a composite curve from these subjects is shown in Fig. 1. In the four patients with either moderate or marked obesity, there was slower dis-

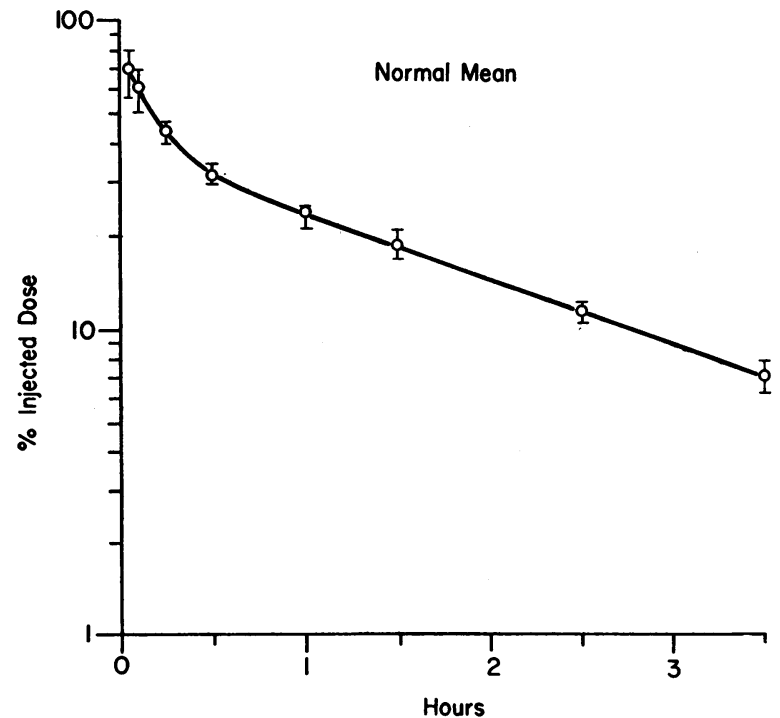

FIGURE 1 Glucose- $6-{ }^{14} \mathrm{C}$ plasma disappearance curve for subjects 1 through 8 . The line indicates the mean value and the bars the spread for each time interval.

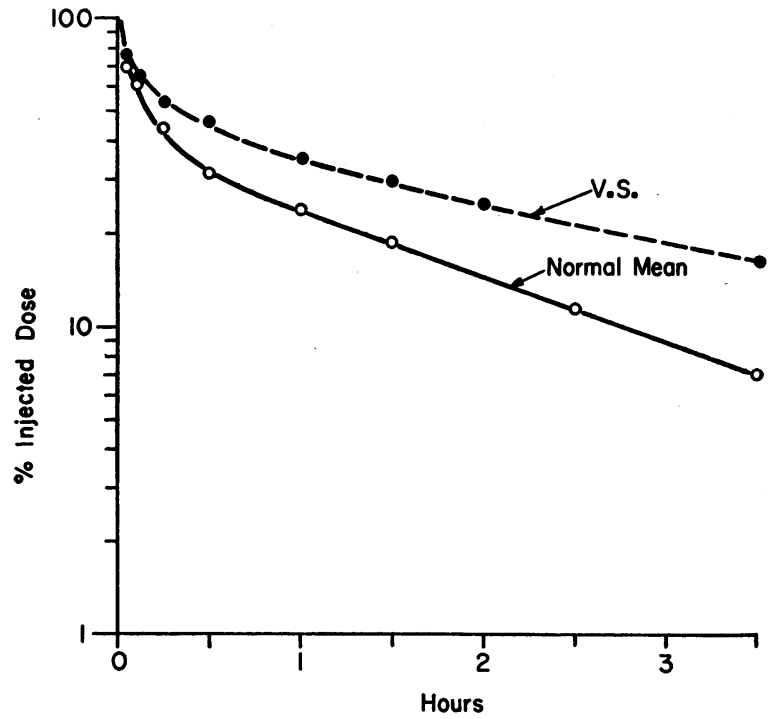

Figure 2 Glucose-6- ${ }^{14} \mathrm{C}$ plasma disappearance curves.

appearance than normal. The most marked variation from the normal curve occurred in V. S. and is shown in Fig. 2. V. S. was the most obese of the subjects studied. It will be noted that all curves are complex, with at least three definable exponents. We make no attempt to assign physiological or anatomical meaning to such components. It does, however, appear that in the great majority of instances the final disappearance rate is manifest by the termination of the study.

Some numerical data obtained from these curves as well as steady-state concentrations of glucose, lactate, and pyruvate are shown in Table II. The final decay rate, $\alpha_{1}$, is shown in order that the effect of obesity on this parameter may be readily seen. The initial volume of distribution of the tracer ${ }^{2}$ is also given. These figures indicate a space slightly larger than blood volume, and again the direct correlation with body weight is seen.

Labeled blood glucose from pyruvate- $3-{ }^{14} C$. The amount of radioactivity found in blood glucose at the various time intervals studied is given in Table III and Figs. 3 and 4. There appear to be two distinct groups of data. In the first, there was a relatively stable amount of radioactive glucose in the blood for the interval studied, and in the other group there was rapid incorporation and decay.

Analysis of the data. The analysis of the data is based on the premise that both pyruvate and lactate uptake by the liver occur in the fasting state. These substances may

\footnotetext{
${ }^{2}$ This value was obtained by extrapolation of $2,3,4$, and $5 \mathrm{~min}$ values of per cent injected dose per cc back to 0 time and substitution in the equation $Q_{0} / Q(t)$ where $Q_{0}$ is injected radioactivity, $Q(t)$ is the radioactivity in glucose per cc blood at $t=0$. Usually a single logarithmic decay was seen during this time with a half-time of about $5 \mathrm{~min}$.
} 
TABLE II

Data from Glucose-6-14 C Disappearance Curves

\begin{tabular}{|c|c|c|c|c|c|}
\hline \multirow[b]{2}{*}{ Patient } & & \multirow{2}{*}{$\begin{array}{c}\text { Initial } \\
\text { volume } \\
\text { of distri- } \\
\text { bution }\end{array}$} & \multicolumn{3}{|c|}{$\begin{array}{l}\text { Steady-state blood } \\
\text { concentration }\end{array}$} \\
\hline & & & Sugar & $\begin{array}{l}\text { Pyru- } \\
\text { vate }\end{array}$ & $\begin{array}{l}\text { Lac- } \\
\text { tate }\end{array}$ \\
\hline & $\alpha_{1}^{*}$ & liters & $\begin{array}{c}m g / 100 \\
m l\end{array}$ & $\begin{array}{c}m g / 100 \\
m l\end{array}$ & $\begin{array}{c}m g / 100 \\
m l\end{array}$ \\
\hline R. N. & 0.433 & 6.7 & 80 & 0.72 & 9.5 \\
\hline J. F. & 0.43 & 6.9 & 85 & 0.65 & 6.2 \\
\hline P. L. & 0.43 & 7.6 & 80 & 0.59 & 5.2 \\
\hline K. H. & 0.493 & 5.9 & 83 & 0.38 & 3.2 \\
\hline D. P. & 0.46 & 6.3 & 83 & 0.54 & 6.0 \\
\hline C. V. & 0.41 & 6.9 & 79 & 0.67 & 7.2 \\
\hline E. C. & 0.51 & 9.2 & 72 & & \\
\hline D. R. & 0.5 & 6.0 & 82 & 1.0 & 10.5 \\
\hline J. S. & 0.28 & 8.8 & 85 & 0.91 & 6.7 \\
\hline M. 0 . & 0.295 & 8.7 & 83 & 0.68 & 6.4 \\
\hline N. S. & & $10.0 \ddagger$ & 85 & & \\
\hline V. S. & 0.28 & 13.8 & 90 & & \\
\hline
\end{tabular}

* $\alpha_{1}$ is the final disappearance rate as seen on glucose disappearance curve.

¥ Since glucose-6-14 $\mathrm{C}$ was not given to this patient, the initial volume of distribution was estimated from curves on similarly obese subjects. The irreversible disposal rate is also estimated from the same curves.

be incorporated into glucose there and then released into the blood. The glucose then disappears from the circulation by a characteristic and determined disappearance rate. We have then, in the incorporation of labeled pyruvate to glucose curve, a combination of the rate process of gluconeogenesis from lactate and pyruvate, as well as

TABLE III

Per cent of Injected Dose of Pyruvate-3-14C in Blood Glucose*

\begin{tabular}{|c|c|c|c|c|c|c|c|c|}
\hline \multirow[b]{2}{*}{ Patient } & \multicolumn{8}{|c|}{ Min } \\
\hline & 5 & 15 & 30 & 60 & 90 & 120 & 150 & 180 \\
\hline R. N. & & 1.9 & 2.0 & 2.2 & 2.1 & 2.1 & & 2.0 \\
\hline J. F. & & 2.2 & 1.8 & 1.7 & 1.4 & & 1.2 & \\
\hline P. L. & 2.0 & 3.0 & 3.1 & 3.0 & 3.0 & & 2.7 & \\
\hline K. H. & & 10.5 & 8.9 & 7.5 & 6.5 & & 3.6 & \\
\hline D. P. & & 8.7 & 7.0 & 5.8 & 4.7 & & 3.0 & \\
\hline C. V. & & 14.3 & 12.8 & 12.0 & 9.8 & & 6.8 & \\
\hline E. C. & & 9.8 & 10.2 & 9.0 & 7.8 & 7.0 & & 4.8 \\
\hline D. R. & & & & 1.5 & 1.8 & & 1.3 & \\
\hline J. S. & & 3.8 & 4.9 & 6.3 & & 5.6 & & 5.0 \\
\hline M. O. & 0.8 & 1.5 & 1.6 & 1.9 & 1.8 & & 1.5 & \\
\hline N. S. & 2.6 & 3.4 & 4.2 & 4.1 & 3.7 & & 3.2 & \\
\hline V. S. & & & 5.3 & 5.9 & 6.6 & & 5.5 & \\
\hline
\end{tabular}

* This is calculated as the dpm in the glucose of $1 \mathrm{ml}$ of blood times the initial volume of distribution of glucose divided by the injected dose of tracer.

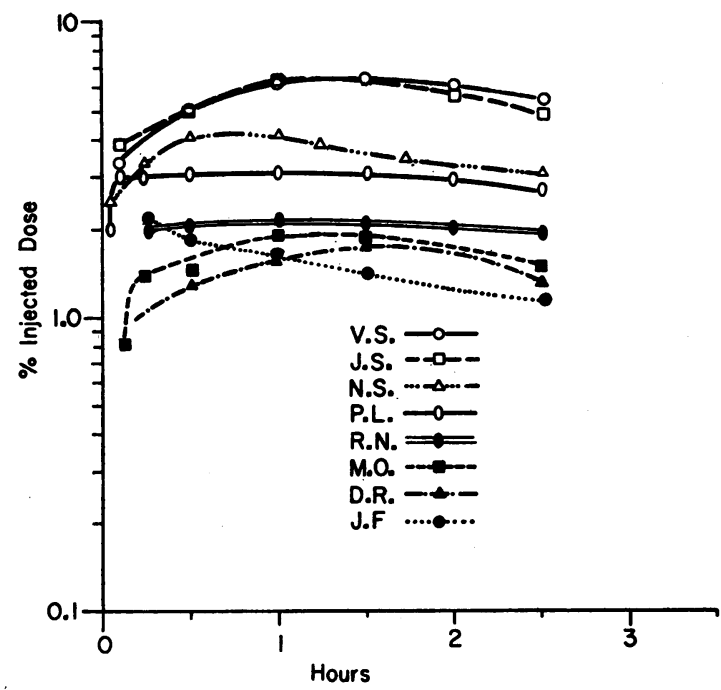

FIgURE 3 Radioactivity- in blood glucose after pyruvate$3{ }^{14} \mathrm{C}$ injection in 8 of the 12 subjects (Nos. $1,2,3,8,9,10$, 11 , and 12). Note the relatively stable radioactivity over the $2 \frac{1}{2} \mathrm{hr}$ interval.

the disappearance of blood glucose. Mathematical description of this sequence of events may be explained as follows :

Let $P_{11}(t)$ equal the amount of radioactivity in any state $j$ at any time $t$ when unit radioactivity was injected at state $i$ at time 0 . Let $S_{1 j}(t)$ equal the probability density for first arrival of a radioactive particle at state $j$ at time $t$, when the injected tracer was in state $i$ at time 0 .

Then, it can be shown that for any two states $i$ and $j$

$$
P_{i j}(t)=S_{i j}(t) * P_{j j}(t),
$$

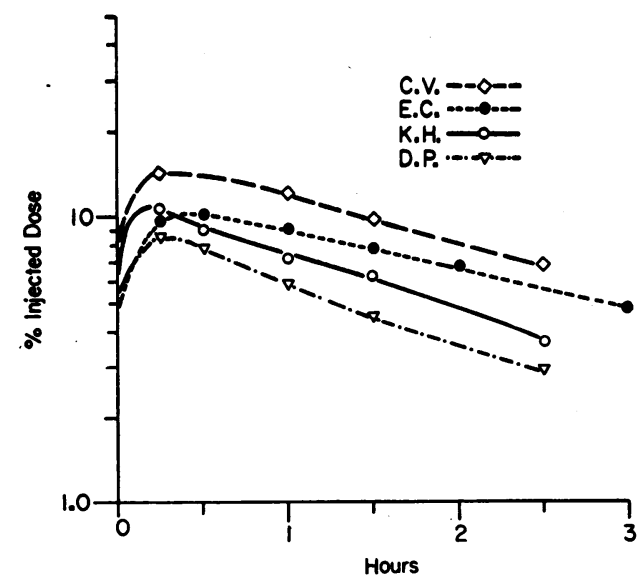

FIGURE 4 Radioactivity in blood glucose after pyruvate-3- ${ }^{11} \mathrm{C}$ injection in subjects $4,5,6$, and 7 . Note the high peak incorporation of about $10 \%$, whereas the group shown in Fig. 3 had a maximum incorporation of $6 \%$. 
where the asterisk denotes convolution, i.e.,

$$
P_{i j}(t)=\int_{0}^{t} S_{i j}\left(t^{\prime}\right) P_{j j}\left(t-t^{\prime}\right) d t^{\prime}
$$

In particular for states $\mathrm{P}$ and $\mathrm{G}$ (pyruvate and glucose), one would have

$$
P_{P G}(t)=S_{P G}(t) * P_{G G}(t) .
$$

Using the above terminology, our curves represent $P_{P G}(t)$ and $P_{G G}(t)$. It is thus possible to solve for $S_{P G}(t)$ by a numerical iteration procedure. It should be noted that this requires no assumptions on the time required for a particle to travel from $P$ to $G$ and, as a matter of fact, defines precisely the arrival rate of radioactive particles. Also repetitive cycling (pyruvate $\rightarrow$ glucose $\rightarrow$ pyruvate $\rightarrow$ glucose, etc.) is allowed in this analysis. The integral $\int_{0}^{\infty} S_{P G}(t) d t$ equals the proportion of blood pyruvate present at any particular time which ultimately enters this pathway and is designated by $\rho^{3}$ If the integral $\int_{0}^{\infty} P_{P G}(t) d t$ can be obtained from the observed data then the relationship below which may be inferred from equation 2 may be used.

$$
\int_{0}^{\infty} P_{P G}(t) d t=\rho \int_{0}^{\infty} P_{G G}(t) d t
$$

where again $\rho$ is the fraction of pyruvate (and lactate) regenerated to glucose.

If we now express the above in its simplest form by the model

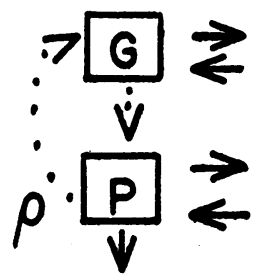

it should be clear that any number of steps may be involved in the transition from $P$ to $G$ and from $G$ to $P$. Reversible exits from $P$ and $G$ may also occur, as well as irreversible exits.

In the majority of the subjects studied here, the curve $P_{P Q}(t)$ is relatively flat, with no final disappearance rate being obvious. There are, however, the four subjects, patients $4,5,6$, and 7 , who show early peak activity and then what appears to be a constant decay rate. In these latter four subjects we have used a second method of analysis described above, namely equation 3. For all

\footnotetext{
'It should be noted that this symbol $\rho$ is not identical to that used by Reichard, Moury, Hochella, Patterson, and Weinhouse (2). In their study, $\rho$ was the proportion of blood glucose which recycled via lactate. If no tracer was lost during the process of glycolysis, the resultant calculations for $\rho$ should theoretically yield the same result.
}

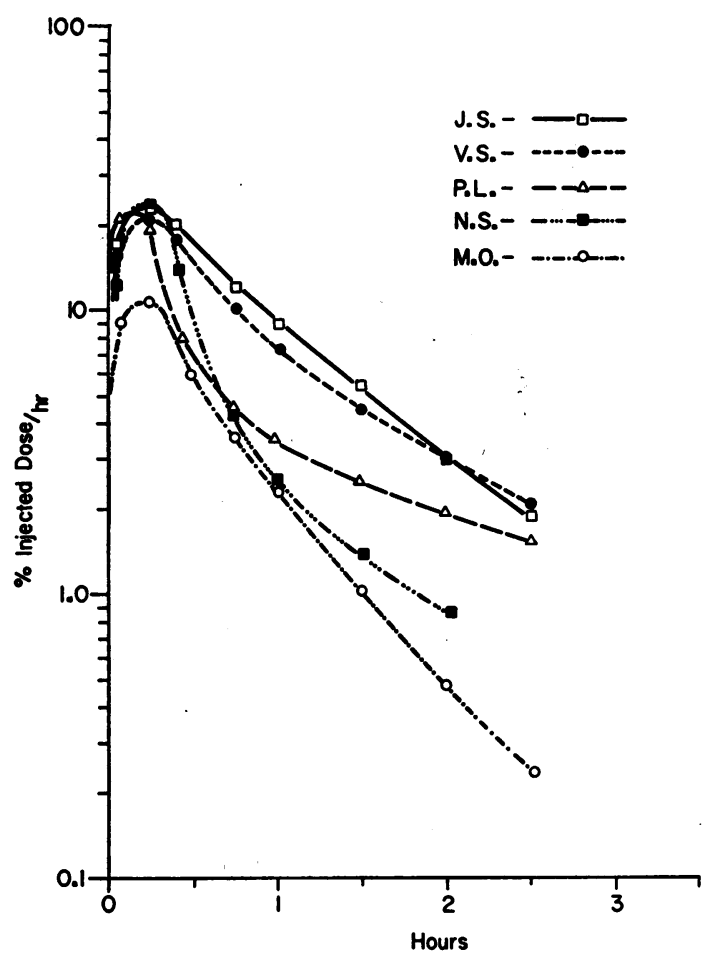

FIgURE 5 First arrival rates of the label from pyruvate-

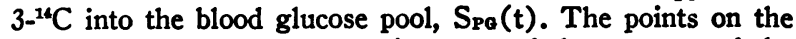
curve are instantaneous rates in terms of the per cent of the dose per hour which arrives at $G$ at time $t$. The data were obtained by deconvolution of $P_{P G}(t)$ with $P_{G G}(t)$ and are representative of the group of subjects $(1,2,3$, and 8 through 12).

other subjects and indeed for confirmation of consistency, deconvolution of $\mathrm{P}_{\mathrm{Pa}}$ and $\mathrm{P}_{\mathrm{ag}}$ has been carried out to solve for Spa. Characteristic curves for $S_{p a}(t)$ in both groups of subjects are presented in Figs. 5 and 6 . It is clear that these curves which represent the first arrival rate from pyruvate into blood glucose are timedependent. Although peak arrival occurs usually within the first $15 \mathrm{~min}$, almost all show some tailing effect in later hours. It seems clear that either a certain proportion of the pyruvate is sequestered and unavailable for early glucose resynthesis or that two different pathways for Cori recycling occur, one with relative rapidity, and the other one slower. The slow regeneration not allowed for in other methods (2) might result in calculation of a greater percentage recycled at long time intervals than at short time intervals. Our calculations for $\rho$ or the fraction of blood pyruvate which is returned to blood glucose for the different subjects are found in the first column of Table IV. It is impossible to differentiate the per cent recycled in the obese or diabetic subjects from the normal ones. One subject stands out because of the high per cent of recycling, namely $\mathrm{C}$. V. The results in 
this subject as well as several others with relatively high values will be discussed later.

Quantitation of Cori cycle. All our calculations so far are in terms of the fraction of pyruvate which recycles to glucose. We should like to convert this to actual grams per unit time and thus determine the quantitative importance of lactate and pyruvate in the over-all process of gluconeogenesis. A precise model of the total system under study is necessary for this. Fig. 7 shows the model, the solution of which is given in the Appendix. It should be emphasized that the analysis is valid for any number of intervening steps where the dotted lines occur in the figure and that the solution is dependent on the use of transfer functions to describe such processes. The only instantaneous transitions used are those designated by $\lambda_{1}$ 's. In what follows, $\mathrm{Pa}(\infty)$ will denote the amount of cold glucose in blood in the steady state. Similarly $I_{m}(\infty)$ will denote the entry rate of cold material into the system in the steady state. It should be remembered that double subscript entities such as $P_{1 j}(t)$ are normalized to unit amount initially in state $i$ and presuppose smallness and distinguishability against the cold steady background, as for the trace material by which they are measured.

Reference to Fig. 7 shows that the material leaving $G$ for $P, P_{G}(\infty) \lambda_{G P}$, is related to the input flow rate,

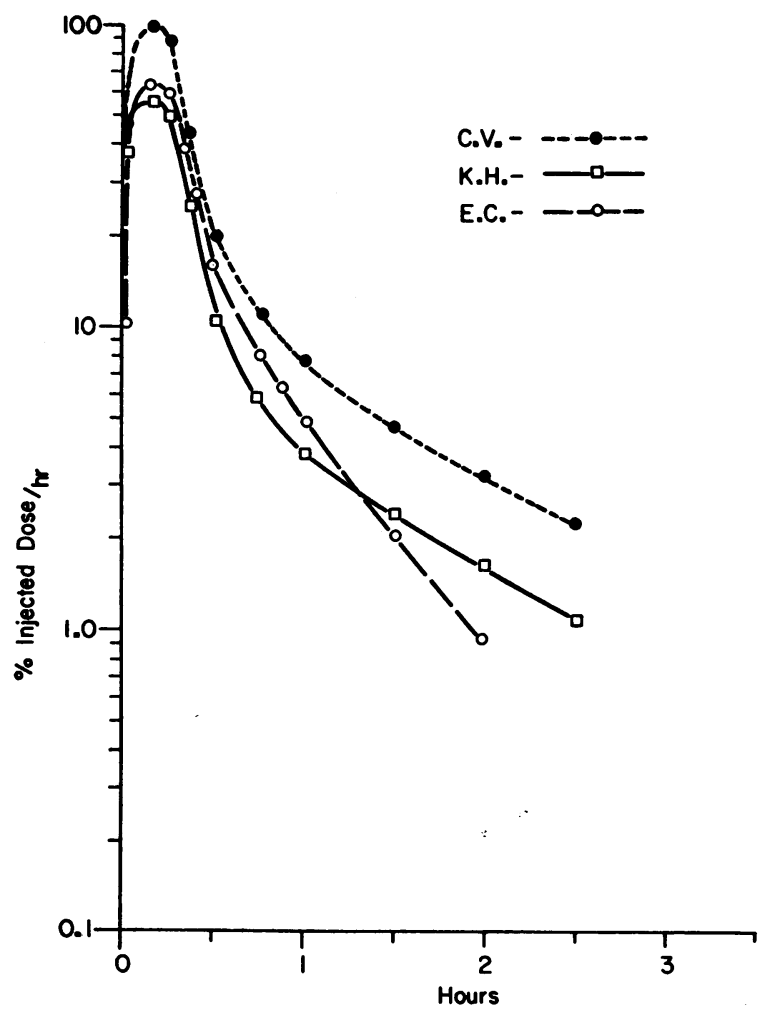

FIGURE 6 First arrival rates representative for subjects 4 through 7.
TABLE IV

Quantitation of Cori Cycle Activity

\begin{tabular}{lcccccc}
\hline Patient & $\rho^{*}$ & $\begin{array}{c}\text { Irreversible } \\
\text { disposal } \\
\text { rate }\end{array}$ & $\begin{array}{c}\text { First disposal } \\
\text { ratef }\end{array}$ & $\begin{array}{c}\text { Pyruvate } \\
\text { and } \\
\text { lactate }\end{array}$ & $\begin{array}{c}\text { Pyruvate } \\
\text { and } \\
\text { lactate }\end{array}$ \\
& & $g / h r$ & $g / h r$ & $\begin{array}{c}m g / k g \\
\text { per } h r\end{array}$ & $g / h r$ & $\begin{array}{c}\text { mg/kg } \\
\text { per } h r\end{array}$ \\
R. N. & 0.16 & 6.08 & 7.3 & 113 & 1.2 & 19 \\
J. F. & 0.11 & 6.61 & 7.4 & 110 & 0.8 & 12 \\
P. L. & 0.13 & 6.84 & 7.9 & 133 & 1.1 & 19 \\
K. H. & 0.29 & 5.54 & 7.8 & 161 & 2.2 & 46 \\
D. P. & 0.27 & 5.96 & 7.7 & 182 & 1.7 & 40 \\
C. V. & 0.55 & 5.3 & 9.6 & 181 & 4.3 & 81 \\
E. C. & 0.24 & 5.77 & 8.5 & 149 & 2.7 & 48 \\
D. R. & 0.07 & 5.54 & 5.9 & 103 & 0.4 & 7 \\
J. S. & 0.23 & 5.62 & 7.3 & 98 & 1.7 & 23 \\
M. O. & 0.09 & 5.9 & 6.5 & 76 & 0.6 & 7 \\
N. S. & 0.16 & 6.96 & 8.3 & 60 & 1.3 & 11 \\
V. S. & 0.21 & 7.46 & 9.5 & 49 & 2.0 & 10 \\
\hline
\end{tabular}

* $\rho$ is fraction of the pyruvate and lactate pool which is ultimately regenerated to glucose.

¥ First disposal rate $=$ amount of glucose which initially leaves the glucose pool for pyruvate; also called "absolute disposal rate" in the text (PGAGP). Amount of pyruvate and lactate recycled to glucose.

$\mathrm{I}_{\boldsymbol{B}}(\infty)$, by the equation $\left.\mathrm{P}_{\mathrm{a}}(\infty) \lambda_{\mathrm{aP}}=\mathrm{I}_{\mathbf{m}}(\infty) / 1-\theta \rho\right)$ (equation A 4), and it follows that the material leaving $P$ which will return to glucose per unit time is related to the input flow rate by the equation $\operatorname{Pr}(\infty) \lambda_{P Q}=$ $\mathrm{I}_{\mathbf{x}}(\infty) \rho \theta /(1-\rho \theta)$ (equation A 6). Thus. if we could calculate I $(\infty)$, use our knowledge of $\rho$, and the reasonable estimate of $\theta \approx 1$, we would obtain the desired milligrams of lactate and pyruvate which are resynthesized to glucose per unit time.

Data which are available to us are $P_{P P}(t)$ and $P_{g a}(t)$ (the radioactivity in blood pyruvate and lactate and that in glucose respectively, when unit amounts of pyruvate$3-{ }^{14} \mathrm{C}$ and glucose $-6{ }^{14} \mathrm{C}$ are injected), as well as the concentration of lactate, pyruvate, and glucose in blood. This theoretically allows us the information of $\mathrm{Pa} / \pi_{G G}$, and $\mathrm{P}_{\mathbf{P}} / \pi_{\mathbf{P P}}$, or the integrated specific activity disappearance curves of glucose and pyruvate. The relationship of such data to the "irreversible disposal rate" $(7,8)$ or equivalently "the input rate, $I_{\mathbb{x}}(\infty)$," is as follows:

$$
\begin{aligned}
& \mathrm{I}_{\mathbf{E}}(\infty)=\mathrm{P}_{\mathrm{G}}(\infty) / \pi_{\mathrm{GG}} \\
& \mathrm{I}_{\mathbf{E}}(\infty)=\mathrm{P} \mathbf{P}(\infty) / \pi_{\mathrm{PP}} \theta_{\mathrm{GP}} .
\end{aligned}
$$

Inspection of the pyruvate disappearance curves as seen in Fig. 8 shows that integration of those curves will be inaccurate. The final disappearance rate becomes manifest only with low activity specimens and appears

4 We employ the notation

$$
\begin{aligned}
& \pi_{G G}(s)=\int_{0}^{\infty} P_{G G}(t) e^{-s t} d t \\
& \pi_{G G}(0)=\int_{0}^{\infty} P_{G G}(t) d t=\pi_{G G} .
\end{aligned}
$$


to be very slow. Thus, extrapolation to infinity carries large potential error. However, referral back to Fig. 1 shows that the glucose curve had a well defined final disappearance rate. $I_{m}$ or the irreversible disappearance rates as calculated from these curves and from the formula above gives the values shown in Table IV.

We may now use equation $4 \mathrm{~A}$ as stated above to obtain the amount of glucose entering the pyruvate pathway $\left(\mathrm{P}_{\mathrm{G}} \lambda_{G P}\right)$, if we assume no irreversible loss between $\mathrm{G}$ and $\mathrm{P}$ (i.e. if $\theta \approx 1$ ). Such values are also recorded in Table IV and are called "first disposal rate" or "absolute removal rate" from the glucose pool. The grams of lactate and pyruvate recycled are then determined by equation A 6 or $P_{P} \lambda_{P G}=I_{E} \rho \theta /(1-\rho \theta)$. Such figures for recycling both in terms of $\mathrm{g} / \mathrm{hr}$ and $\mathrm{g} / \mathrm{kg}$ per $\mathrm{hr}$ are found in the last two columns of Table IV.

It will be noted that the assumption of little irreversible loss during glycolysis (i.e. $\theta \approx 1$ ) appears an important one; yet a $20 \%$ loss of label would give only a $4 \%$ error in our "absolute removal rate" of glucose and a $6 \%$ error in the recycled lactate and pyruvate. Physiological considerations make it highly unlikely that much glucose is irreversibly lost during glycolysis in the fasting state since storage of glycogen or formation of pentose and glycerol should be minimal under this condition.

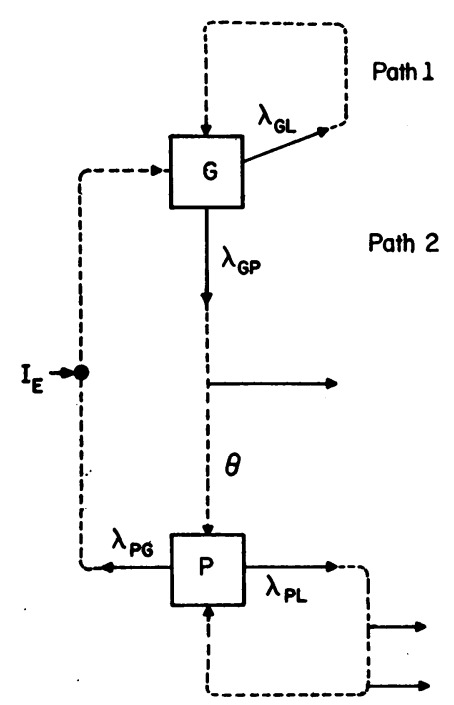

FIGURE 7 State G, glucose in blood and rapidly equilibrating sites. State $\mathrm{P}$, pyruvate and lactate in blood and rapidly equilibrating cellular sites. $I_{\mathbb{w}}$, input flow rate from outside the system. $\theta$, fraction of glucose leaving $G$ which reaches $P$. $\rho$, fraction of pyruvate in $P$ which will eventually return to $G$. $\lambda_{11}$, fractional turnover rate from compartment $i$ to $j$. Arrows, which leave the system, denote losses.

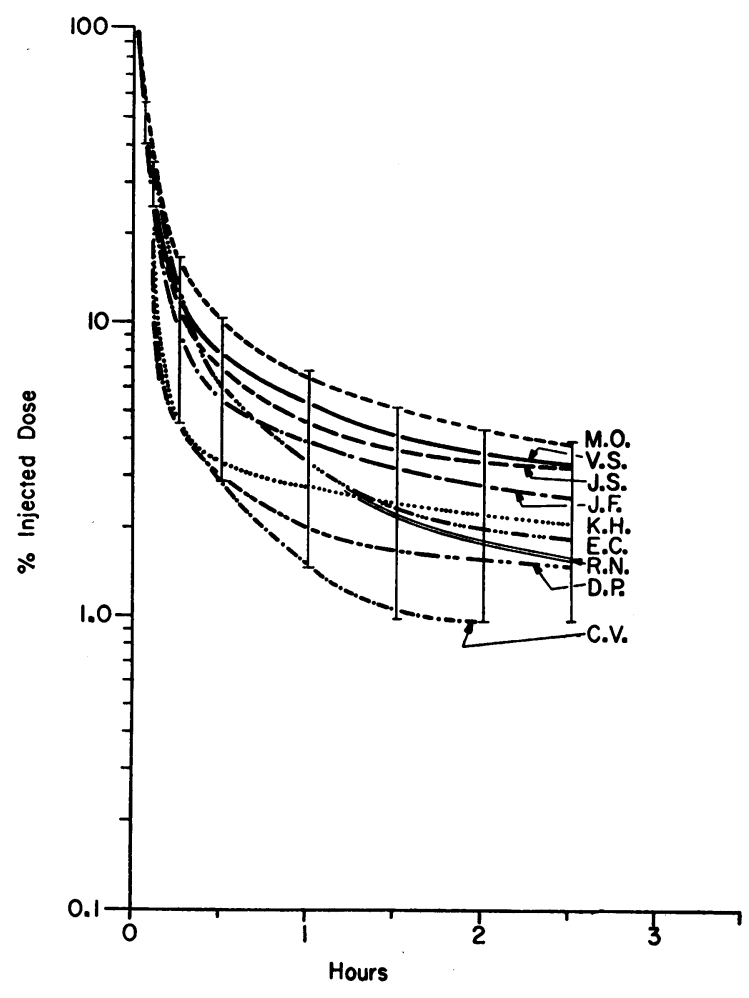

FIGURE 8 Blood pyruvate disappearance curves for 9 of the 12 patients studied.

\section{DISCUSSION}

In the three studies thus far reported on Cori cycle activity in human beings $(2-4)$, the range of values for normal people after an overnight fast is quite large. Reichard's (2) original paper includes four normal individuals where the per cent of glucose recycled was from 12 to 20 , with an average of $26 \mathrm{mg} / \mathrm{kg}$ per hr regenerated. The study of Cahill et al. (3) of eight normal males after an 8 day fast showed $10-33 \%$ recycling with $6-43 \mathrm{mg} / \mathrm{kg}$ per $\mathrm{hr}$. Kreisberg's (4) normal subjects apparently showed less spread with average value of $15.8 \mathrm{mg} / \mathrm{kg}$ per $\mathrm{hr} \pm 1.0 \mathrm{sEM}$. It is noteworthy that in Myer's study (1) of hepatic glucose production he had four individuals with much higher glucose output than the others and he comments on the fact that these high values might be related to anxiety on the part of these patients. Cahill et al. also report one patient with a very high recycling rate but do not distinguish this subject from the others. In our subjects, 8 out of 12 showed recycling rates varying from 7 to $23 \mathrm{mg} / \mathrm{kg}$ per hr. Included in this group were all the obese and diabetic patients. Our failure to differentiate maturity-onset diabetics from normal individuals does not exclude the possibility that other diabetic subjects with high fasting blood sugars might not show differences. The four subjects well outside this range were all in the group 
originally studied for the possibility of hypoglycemia and in whom our current diagnosis is anxiety state. While we have included them in our control group, since we could not establish abnormality of carbohydrate metabolism by oral and IV glucose tolerance, it now seems possible that this group should be considered separately.

All four subjects with high recycling rates had normal fasting insulin levels as well as a normal response to intravenous and oral glucose loads. Fasting lactate and pyruvate levels were normal. Correlation of high recycling rates with high initial or absolute disposal rates of glucose is obvious, while irreversible disposal does not appear to be increased. Thus, the total amount of glucose produced from all sources is greater than normal but only that portion from glycolysis and regeneration accounts for the increase.

Abnormalities in lactate metabolism have been suggested by several authors in anxiety neurosis and are well summarized by Pitts and McClure (9). While all agree that resting levels are normal, it is postulated that excessive production of lactate under certain circumstances may be present. If indeed such data are confirmed, it is tempting to try to relate these as well as our own findings to cerebral metabolism, since the great majority of glucose uptake in the fasting state is by the brain. Sacks' $(10,11)$ excellent work on brain metabolism would indicate that nearly all glucose uptake as measured with glucose-3- ${ }^{14} \mathrm{C}$ can be accounted for by ${ }^{14} \mathrm{CO}_{3}$ in normal people, implying little net production of lactate by the brain. Confirmation of small AV lactate differences was also obtained. Oxidation and ${ }^{14} \mathrm{CO}_{2}$ production with other labels was considerably slower, and even at $6 \mathrm{hr}$ not all label was accounted for. He interprets that the labeled glucose mixes with a large unlabeled pool in the Krebs cycle. Suggestion of abnormality in patients with mental disease with slower than normal ${ }^{14} \mathrm{CO}$, production is also made (12). Further exploration into this area would be of interest.

Finally we should like to comment about the mode of analysis used to interpret our data. If we view our system in terms of its biochemical steps and physiological and anatomical states, it is apparent that compartmental analysis with a finite number of states and instantaneous transitions, i.e. full incidence matrix, is not possible. We have measured the performance of subsets of the total system, using integrated functions for this purpose. The relationships between the subsets are then described by transfer functions. A definitive model of the sampled compartment of each subset and the sites of loss from the system are the only constraints we have used. We feel that this approach which combines compartmental, integrated, and transfer functions adds far greater scope for biological study than traditional compartmental analysis.

\section{APPENDIX}

Mathematical model for gluconeogenesis. Our model consists of a pool of tagged glucose in blood, designated by $G$, in interaction with the remaining body system assumed to be linear. A second pool of central interest, tagged pyruvate, (P) is part of this body system (Fig. 7). Departures from the pool $G$ either enter at fractional rate $\lambda_{G L}$ a closed (without loss) path, path 1 , which does not involve $P$, or enter path 2 at rate $\lambda_{G P}$ which can only return to $G$ via $P$. Possible losses between $G$ and $P$ and lossy loops from $P$ back to $P$ are indicated in the figure. Also indicated is an input flow rate $I_{E}(t)$ coming from outside the system. When the system is in equilibrium, $I_{E}(t)$ will assume a steady-state value which will balance all losses. Let us imagine that a small part, $I_{E^{*}}(t)$, of this input were tagged so that $I_{E^{*}}(t)$ is the quantity of tagged ${ }^{14} \mathrm{C}$ entering the system per unit time. We employ the following notation.

$\mathrm{P}_{\mathrm{G}}{ }^{*}(\mathrm{t})$ is the total amount of tagged ${ }^{14} \mathrm{C}$ in pool $\mathrm{G}$ at time $\mathrm{t}$. $\mathrm{S}_{\mathrm{GPG}}{ }^{\dagger}(\tau) \mathrm{d} \tau$ is that fraction of unit amount of trace entering path 2 at $t=t_{1}$ which returns subsequently to $G$ for the first time in the interval $\left(t_{1}+\tau, t_{1}+\tau+d \tau\right)$. Thus $\mathrm{S}_{\mathrm{GPG}}{ }^{\dagger}(\tau)$ is a probability density for elapsed time $\tau$ since time of entry $t_{1}$ into path 2.5

$\mathrm{S}_{\mathrm{GLG}}{ }^{\dagger}(\tau) \mathrm{d} \tau$ is that fraction of unit amount of trace entering path 1 at $t=t_{1}$ which returns subsequently to $G$ for the first time in $\left(t_{1}+\tau, t_{1}+\tau+d \tau\right)$.

We suppose that no tagged material is present before $t=0$. Then $\mathrm{P}_{\mathrm{G}}{ }^{*}(\mathrm{t})$ must obey the equation

$$
\begin{aligned}
d_{P_{G}}^{*}(t) / d t & =-\lambda_{G P} P_{G} *(t)+\lambda_{G P} P_{G} *(t) * S_{G P G}{ }^{\dagger}(t) \\
& -\lambda_{G L} P_{G}{ }^{*}(t)+\lambda_{G L} P_{G} *(t) * S_{G L G}{ }^{\dagger}(t) \\
& +I_{E} *(t) * S^{\dagger}{ }_{E G}(t) .
\end{aligned}
$$

The term $-\lambda_{G P} P_{G}{ }^{*}(t)$ represents the rate of loss from $G$ at time $t$ to path 2 and the term

$$
\lambda_{G P} P_{G}^{*}(t) * S_{G P G}{ }^{\dagger}(t)=\int_{0}^{t} \lambda_{G P} P_{G}^{*}(\tau) S_{G P G}{ }^{\dagger}(t-\tau) d \tau
$$

represents the rate of return to $G$ at time $t$ from path 2 . Let Laplace transforms be designated by lower case Greek letters, e.g., $\mathrm{L}\left\{\mathrm{P}_{\mathrm{G}} *(\mathrm{t})\right\}=\pi_{\mathrm{G}} *(\mathrm{~s})$. Then transformation of $\mathrm{A} 1$ gives

$$
\begin{aligned}
s \pi_{\mathrm{G}}^{*}(\mathrm{~s}) & -\mathrm{P}_{\mathrm{G}}{ }^{*}(0)=-\lambda_{\mathrm{GP} \pi_{\mathrm{G}}}{ }^{*}(\mathrm{~s})\left\{1-\sigma_{\mathrm{GPG}}{ }^{\dagger}(\mathrm{s})\right\} \\
& -\lambda_{\mathrm{GL}} \pi_{\mathrm{G}}{ }^{*}(\mathrm{~s})\left\{1-\sigma_{\mathrm{GLG}}{ }^{\dagger}(\mathrm{s})\right\}+\mathrm{i}_{\mathrm{E}}^{*}(\mathrm{~s}) \sigma^{\dagger}{ }_{\mathrm{EG}}(\mathrm{S}) .
\end{aligned}
$$

Now suppose that $I_{E}{ }^{*}(t)$ assumes a steady value as $t \rightarrow \infty$. If we multiply A 3 by s, permit $s$ to go to zero, and note that

$$
\begin{aligned}
& \lim _{t \rightarrow \infty} P_{G^{*}}(t)=\lim _{s \rightarrow 0+} s \pi^{*}(s)=P_{G} *(\infty), \\
& \lim _{t \rightarrow \infty} I_{E}^{*}(t)=\lim _{s \rightarrow 0^{+}} \operatorname{si}_{E}^{*}(s)=I_{E}^{*}(\infty),
\end{aligned}
$$

and

we find

$$
\sigma_{\mathrm{GLG}}{ }^{\dagger}(0)=1 \text { and } \sigma_{\mathrm{EG}}^{\dagger}(0)=1 \text {, }
$$

$$
\begin{aligned}
& \lambda_{\mathrm{GP}} \mathrm{P}_{\mathrm{G}}^{*}(\infty)=\mathrm{I}_{\mathrm{E}}^{*}(\infty) /\left\{1-\sigma_{\mathrm{GPG}}^{\dagger}(0)\right\} \\
& \text { or } \mathrm{I}_{\mathrm{GP}}{ }^{*}(\infty)=\mathrm{I}_{\mathrm{E}}^{*}(\infty) /\{1-\rho \theta\} .
\end{aligned}
$$

- It is customary in probability theory for $\mathrm{S}_{\mathrm{AB}}(\tau)$ to denote the density of passage time from being in state $A$ to first arrival at $B$. The random variable $\tau$ then includes the time spent sitting in state $A$. For us $S^{\dagger}(\tau)$ denotes the path time density, with dwell time in state A excluded. 
For $\sigma_{\mathrm{GPG}}{ }^{\dagger}(0)=\int_{0}^{\infty} \mathrm{S}_{\mathrm{GPG}}{ }^{\dagger}(\tau) \mathrm{d} \tau$ is just the fraction of trace entering path 2 which ultimately returns to $G$. If we denote the fraction entering path 2 which reaches pool $\mathrm{P}$ by $\theta$ and the ratio of those entering $P$ which reach $G$ ultimately by $\rho$, we have

$$
\sigma_{\mathrm{GPG}}{ }^{\dagger}(0)=\theta \rho .
$$

In $\mathrm{A} 4, \lambda_{\mathrm{GP}} \mathrm{P}_{\mathrm{G}}{ }^{*}(\infty)$ is the steady-state flow $\mathrm{I}_{\mathrm{GP}}{ }^{*}(\infty)$ into path 2 . Then the steady-state flow

or

$$
\begin{gathered}
\mathrm{I}_{\mathrm{PG}}{ }^{*}(\infty)=\rho \theta \mathrm{I}_{\mathrm{GP}}{ }^{*}(\infty)=\rho \theta \mathrm{I}_{\mathrm{E}}^{*}(\infty) /\{1-\rho \theta\} \\
\mathrm{I}_{\mathrm{PG}}{ }^{*}(\infty) / \mathrm{I}_{\mathrm{E}}^{*}(\infty)=\rho \theta /\{1-\rho \theta\} .
\end{gathered}
$$

We next observe that the behavior of the tagged material is identical in behavior with its cold counterpart. The asterisks denoting tagged character may therefore be removed in equations A 4, A 6, and A 7 and these equations will remain correct. In the text $P_{G}(\infty)$ and $P_{P}(\infty)$ then represent the cold counterparts of $\mathrm{P}_{\mathrm{G}}{ }^{*}(\infty)$ and $\mathrm{P}_{\mathrm{P}}^{*}(\infty)$.

We also have, clearly,

and

$$
\mathrm{P}_{\mathrm{G}} *(\mathrm{t})=\mathrm{I}_{\mathrm{E}} *(\mathrm{t}) * \mathrm{~S}_{\mathrm{EG}}{ }^{\dagger}(\mathrm{t}) * \mathrm{P}_{\mathrm{GG}} *(\mathrm{t})
$$

$$
P_{P}^{*}(t)=I_{E}^{*}(t) * S_{E G}{ }^{\dagger}(t) * S_{G P}(t) * P_{P P} *(t) \text {. }
$$

It follows that

and

$$
\begin{aligned}
& \mathrm{P}_{\mathrm{G}}^{*}(\infty)=\mathrm{I}_{\mathrm{E}}^{*}(\infty) \sigma_{\mathrm{EG}}{ }^{\dagger}(0) \pi_{\mathrm{GG}}{ }^{*}(0) \\
& \mathrm{P}_{\mathrm{P}}^{*}(\infty)=\mathrm{I}_{\mathrm{E}}^{*}(\infty) \sigma_{\mathrm{EG}}{ }^{\dagger}(0) \theta \pi_{\mathrm{PP}}{ }^{*}(0) .
\end{aligned}
$$

Again the asterisks may be removed, and since $\sigma_{\mathrm{EG}}{ }^{\dagger}(0)=1$, we obtain equations 4 and 5 of the text.

\section{ACKNOWLEDGMENTS}

This study was supported by Public Health Service Research Grant CA 07123 and by Public Health Service Research Grant FR 00044 from the Division of Research Facilities and Resources, National Institutes of Health.

\section{REFERENCES}

1. Myers, J. D. 1950. Net splanchnic glucose production in normal man and in various disease states. J. Clin. Invest. 29: 1421 .

2. Reichard, G. A., Jr., N. F. Moury, Jr., N. J. Hochella, A. L. Patterson, and S. Weinhouse. 1963. Quantitative estimation of the Cori cycle in the human. J. Biol. Chem. 238: 495.

3. Cahill, G. F., Jr., M. G. Herrera, A. P. Morgan, J. S. Soeldner, J. Steinke, P. L. Levy, G. A. Reichard, Jr., and D. M. Kipnis. 1966. Hormone-fuel interrelationships during fasting. J. Clin. Invest. 45: 1751.

4. Kreisberg, R. A. 1968. Glucose metabolism in normal and obese subjects. Diabetes. 17: 481.

5. Blair, A., and S. Segal. 1960. The isolation of blood glucose as potassium gluconate. J. Lab. Clin. Med. 55: 959.

6. Waterhouse, C., and J. H. Kemperman. 1966. Changes in oxidative metabolism with glucose ingestion. $J$. Lab. Clin. Med. 68: 250.

7. Stern, M. P., J. W. Farquhar, A. Silvers, and G. M. Reaven. 1968. Insulin delivery rate into plasma in normal and diabetic subjects. J. Clin. Invest. 47: 1947.

8. Waterhouse, C., N. Baker, and H. Rostami. 1969. Effect of glucose ingestion on the metabolism of free fatty acids in human subjects. J. Lipid Res. 10: 487.

9. Pitts, F. N., Jr., and J. N. McClure, Jr. 1967. Lactate metabolism in anxiety neurosis. N. Engl. J. Med. 277: 1329.

10. Sacks, W. 1957. Cerebral metabolism of isotopic glucose in normal human subjects. J. Appl. Physiol. 10: 37.

11. Sacks, W. 1965. Cerebral metabolism of doubly labeled glucose in humans in vivo. J. Appl. Physiol. 20: 117.

12. Sacks, W. 1961. Cerebral metabolism of glucose-3-C $C^{\mathbf{1 4}}$, pyruvate-1-C and lactate-1-C $\mathrm{C}^{\mathbf{1 4}}$ in mental disease. $J$. Appl. Physiol. 16: 175. 